\title{
The Friedrichs-Model with fermion-boson couplings II.
}

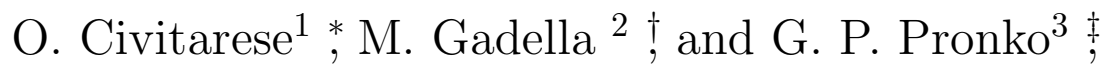 \\ ${ }^{1}$ Departamento de Física, Universidad Nacional de La Plata, \\ c.c. 67 1900, La Plata, Argentina. \\ ${ }^{2}$ Departamento de Física Teórica, Facultad de Ciencias, E-47011, Valladolid, Spain \\ ${ }^{3}$ Institute for High Energy Physics, Protvino, 142284,Moscow Region. Russia \\ and Institute of Nuclear Physics, N.C.S. "Demokritos".
}

November 12, 2018

\begin{abstract}
In this work we present a formal solution of the extended version of the Friedrichs Model. The Hamiltonian consists of discrete and continuum bosonic states, which are coupled to fermions. The simultaneous treatment of the couplings of the fermions with the discrete and continuous sectors of the bosonic degrees of freedom leads to a system of coupled equations, whose solutions are found by applying standard methods of representation of bound and resonant states.
\end{abstract}

PACS: $03.65 \mathrm{Nk}, 03.70+\mathrm{k}$

\footnotetext{
*e-mail: civitare@fisica.unlp.edu.ar

$\dagger^{\dagger}$ e-mail: gadella@fta.uva.es

‡e-mail: pronko@ihep.ru
} 


\section{Introduction}

The Friedrichs model [1] describes the coupling of discrete and continuum sectors of a boson Hamiltonian. The model has the advantage of being exactly solvable and it has been applied to a variety of systems of physical interest [2]. The mathematical aspects of the solutions which are based in the use of rigged Hilbert spaces have been introduced in [3. A distinctive feature of the model is the appearance, among the solutions, of Gamow states [4, 5, 6]. It is the only completely solvable model which describes resonance phenomena [3] in a broad context. Generally speaking, the solutions show the importance of the discrete-continuum couplings, a fact that has been overlooked for quite a while and that certainly has crucial physical consequences. In fact, the need for the inclusion of bound as well as resonant states in the description of composite systems has been demonstrated (see [7] and references quoted therein) in connection with the analysis of data obtained in experiments measuring the decay of clusters. The properties of the standard version of the Friedrichs model, in connection with Gamow resonances, have been revisited in [5]. More sophisticated versions of the Friedrichs model have been presented in [6, 8, 10. Along the standard discrete-continuous couplings for bosons, one has to consider also the boson-boson couplings [2] and fermi-boson couplings [6]. In a recent paper [6], we have proposed an extended version of the model to accommodate for fermion-boson couplings. The structure of the extended Hamiltonian is inspired in standard field theory models of the coupling between fermion and boson fields [11, 12]. In a previous publication [6], hereafter referred to as I, the feasibility of the extended model was discussed and its solution were explored within a schematic model situation. By considering a very reduced space consisting of one discrete fermion state in addition to the bosonic degrees of freedom, it was found a resonant behavior also in the fermion sector, as expected. In this paper, we focus our attention in the analysis of the solution within a more general framework. We base our method on the use of the resolvent for the characteristic coupled equations which describes the amplitude of the fermionic solution. The coupled equations are cast in a form which very much resembles the T-matrix formalism [13], which is very convenient for finding out the resonance behavior.

The present paper is organized as follows: In section 2, we review the essentials of the extended Friedrichs model, which in full detail has been introduced in [6], and focus our attention on the elements needed for the 
present discussion. In section 3, we analyze the structure of the general solution which has been found by applying the T-matrix formalism. Finally, we draw our conclusions on section 4 .

\section{Formalism}

We shall begin with a review of the basic ideas of the Friedrichs Model [1, 5]. Details about the formalism concerning the standard Friedrichs model can be found in [14]-[16] and references quoted therein.

The simplest form of the Friedrichs model [1] includes a free Hamiltonian $H_{0}$ with a real positive continuous spectrum coinciding with the positive semiaxis, $\omega>0$, and a positive discrete eigenvalue, $\omega_{0}>0$. An interaction is acting between the continuous and discrete parts of $H_{0}$ by means of a potential $V$. As a result of the action of $V$, the bound state of $H_{0}$ is dissolved in the continuous and a resonance is produced. The spectrum of the total Hamiltonian $H=H_{0}+\lambda V$, where $\lambda$ is a real coupling constant, is purely continuous and coincides with the real positive semiaxis as well [1, 3].

As shown in I, poles of the solutions appear into complex conjugate pairs, each of them represents a resonance. Then, Gamow vectors are solutions of the eigenvalue equation [2, 4],

$$
(H-x) \Psi(x)=0 .
$$

with the total Hamiltonian $H$, whose eigenvalues coincide with resonance poles [3, 16].

\subsection{The extended Friedrichs Model.}

The present version of the Friedrichs model, as an extension of the standard Friedrichs model, includes [6]:

i) The unperturbed fermion and boson Hamiltonian $H_{I}$

$$
H_{I}=\omega_{0}|1\rangle\left\langle 1\left|+\int_{0}^{\infty} d \omega \omega\right| \omega\right\rangle\left\langle\omega\left|+\sum_{k} c_{k}\right| k\right\rangle\langle k|,
$$

where the index $k$ runs out the set of Fermion kets $|k\rangle$.

ii) The interaction between fermions, $|k\rangle$, and the discrete boson, $|1\rangle$, Hamiltonian $H_{I I}$ : 


$$
H_{I I}=\sum_{k, l}\left[h_{k, l}|k, 1\rangle\left\langle l\left|+h_{k, l}^{*}\right| l\right\rangle\langle k, 1|\right]
$$

iii) The interaction between fermions and the boson field, $\{|\omega\rangle\}, H_{I I I}$ :

$$
H_{I I I}=\sum_{k, l} \int_{0}^{\infty} d \omega\left[f_{k, l}(\omega)|k, \omega\rangle\left\langle l\left|+f_{k, l}^{*}(\omega)\right| l\right\rangle\langle k, \omega|\right] .
$$

The standard Friedrichs model includes the boson-boson coupling $V$ [3]. This coupling $V$ can be generalized to include fermion-boson interactions in the following manner:

$$
H_{I V}=\sum_{k, k^{\prime}} \int_{0}^{\infty} d \omega\left[g_{k k^{\prime}}(\omega)|k, 1\rangle\left\langle k^{\prime}, \omega\left|+g_{k k^{\prime}}^{*}(\omega)\right| k^{\prime}, \omega\right\rangle\langle k, 1|\right] .
$$

Alternatively, $H_{I V}$ can be taken as a second order interaction mediated by the fermion state $|k\rangle$, provided that:

$$
\sum_{l} f_{k l}^{*}(\omega) h_{l k^{\prime}}=g_{k k^{\prime}}(\omega) \delta_{k k^{\prime}}=g_{k}(\omega) \delta_{k k^{\prime}},
$$

is fulfilled, as it is customarily done in the field theoretical treatment of fermion-boson couplings [17]. In addition, it is worthy to note that $H_{I V}$ reduces to the standard Friedrichs model interaction, $V$, if $g_{k}(\omega)=g(\omega)$ for all $k$.

To obtain the solution of the eigenvalue problem

$$
(H-E) \Psi(E)=0,
$$

where $H=H_{I}+H_{I I}+H_{I I I}+H_{I V}$, we write $\Psi(E)$ in the form

$$
\Psi(E)=\sum_{k} \varphi_{k}(E)|k\rangle+\sum_{k} \phi_{k, 1}(E)|k, 1\rangle+\sum_{k} \int_{0}^{\infty} d \omega \psi_{k}(E, \omega)|k, \omega\rangle .
$$

Consequently, (7) gives

$$
(H-E) \Psi(E) \equiv \sum_{k} \varphi_{k}(E)\left(c_{k}-E\right)|l\rangle+\sum_{k, l} h_{k l}^{*} \phi_{k, 1}(E)|k\rangle
$$




$$
\begin{aligned}
& +\sum_{k}\left(c_{k}+\omega_{0}-E\right) \phi_{k, 1}(E)|k, 1\rangle+\sum_{k} \int_{0}^{\infty} d \omega \psi_{k}(E, \omega) g_{k}(\omega)|k, 1\rangle \\
& +\sum_{k} \phi_{k, 1}(E) \int_{0}^{\infty} d \omega g_{k}^{*}(\omega)|k, \omega\rangle+\sum_{k} \int_{0}^{\infty} d \omega \psi_{k}(E, \omega)\left(c_{k}+\omega-E\right)|k, \omega\rangle \\
& +\sum_{k, l} \int_{0}^{\infty} d \omega \psi_{k}(E, \omega) f_{k l}^{*}(\omega)|l\rangle+\sum_{i, k} \varphi_{k}(E) h_{i k}|i, 1\rangle \\
& +\sum_{i, k} \int_{0}^{\infty} d \omega \varphi_{k}(E) f_{i k}(\omega)|i, \omega\rangle=0 .
\end{aligned}
$$

Due to the linear independence of the vectors $|k\rangle,|k, 1\rangle$, and $|k, \omega\rangle$, the above equation can be written as a system of the following three coupled equations:

$$
\begin{gathered}
\varphi_{k}(E)\left(c_{k}-E\right)+\sum_{l} h_{l k}^{*} \phi_{l 1}(E)+\sum_{l} \int_{0}^{\infty} d \omega \psi_{l}(E, \omega) f_{l k}^{*}(\omega)=0 \\
\sum_{l} \varphi_{l}(E) h_{k l}+\left(c_{k}+\omega_{0}-E\right) \phi_{k 1}(E)+\int_{0}^{\infty} d \omega \psi_{k}(E, \omega) g_{k}(\omega)=0 \\
\psi_{k}(E, \omega)\left(c_{k}+\omega-E\right)+\sum_{l} \varphi_{l}(E) f_{k l}(\omega)+\phi_{k 1}(E) g_{k}^{*}(\omega)=0
\end{gathered}
$$

for $|k\rangle,|k, 1\rangle$, and $|k, \omega\rangle$, respectively. using (10) and (11) in (12), we obtain the following equation:

$$
\psi_{k}(E, \omega)=c \delta\left(c_{k}-\omega-E\right)-\sum_{l} \frac{\varphi_{l}(E) f_{k l}(\omega)}{c_{k}-\omega-E}-\frac{\phi_{k 1}(E) g_{k}^{*}(\omega)}{c_{k}-\omega-E}
$$

Consequently, if $\psi_{k}(E, \omega)$ is reinserted into (10) and (11), it results a system of infinite coupled equations. The formal properties of its solutions becomes obvious at glance: i.) the resonant behavior of the fermionic solutions results from the coupling to the continuum-continuum boson sector; ii.) the resonant boson sector is mostly unaffected by the fermion sector, since the latter solely depends on $V$.

The solutions of the system depend on the choices of the form factors $h_{k l}$, $f_{k l}(\omega)$ and $g_{k}(\omega)$. After a rearrangement, (10) and (11) yields: 


$$
\begin{array}{r}
{\left[\left(c_{k}-E\right) \delta_{k m}-\sum_{m} A_{k m}(E)\right] \varphi_{m}(E)} \\
+\sum_{m}\left(h_{m k}^{*}-B_{k m}(E)\right) \phi_{m 1}(E)=-c \sum_{m} f_{m k}^{*}\left(E-c_{m}\right)
\end{array}
$$

and

$$
\begin{aligned}
& \sum_{l}\left[h_{k l}-\widetilde{B}_{k l}(E)\right] \varphi_{l}(E) \\
& +\left(c_{k}+\omega_{0}-E-C_{k}(E)\right) \phi_{k 1}(E)=-c g_{k}\left(E-c_{k}\right) .
\end{aligned}
$$

Note that the functions, $A_{k m}(E), B_{k m}(E), \widetilde{B}_{k l}(E)$ and $C_{k}(E)$ are explicit functions of the form factors. These functions have been given in I, (see Equations 32-35 of [6]). The factor $c$ in (15) is an irrelevant constant.

\section{Choice of form factors.}

In I, we have shown that the system of coupled equations which determine the amplitudes $\varphi_{k}(E), \phi_{l 1}(E)$ and $\psi_{k}(E, \omega)$ can be solved under certain simplifications like the assumption that the form factors do not depend on the fermionic indices and/or that the fermionic subspace be severely reduced. Here, we propose a more general setting for finding the solution, which consists of the study of the separate couplings of the fermions with the discrete and continuous bosons. For the form factors, we here propose the following choice:

$$
f_{l k}(\omega)=\alpha_{l} \alpha_{k}^{*} \rho(\omega), \quad h_{l k}=\beta_{l} \beta_{k}^{*}, \quad g_{k}(\omega)=0,
$$

This choice does not contradict (6) because we are considering the couplings (21), (3) and (44) as independent couplings, of which (6) is a special case only. Here, $\rho(\omega)$ is an unspecified function, whose explicit structure is not needed for the present discussion. In actual calculations it is defined by the model Hamiltonian. Thus, with this choice of the form factors, Equation (15) reads: 


$$
\phi_{k 1}(E)=-\sum_{s} \frac{\varphi_{s}(E) h_{k s}}{c_{k}-\omega_{0}-E} .
$$

Then, Equations (32-35) in I become

$$
\begin{array}{r}
A_{k m}(E)=\int_{0}^{\infty} d \omega \sum_{l} \frac{\alpha_{l}^{*} \alpha_{k} \alpha_{l} \alpha_{m}^{*}|\rho|^{2}}{c_{l}+\omega-E} \\
=\alpha_{k} \alpha_{m}^{*} \int_{0}^{\infty} d \omega \sum_{l} \frac{\alpha_{l}^{*} \alpha_{l}|\rho|^{2}}{c_{l}+\omega-E}=\alpha_{k} \alpha_{m}^{*} A(E) . \\
B_{k m}=0, \quad \widetilde{B}_{k m}=0, \quad C_{k m}=0 .
\end{array}
$$

Note that (18) defines $A(E)$. This yields the following form for (14):

$$
\begin{array}{r}
\sum_{m}\left[\left(c_{k}-E\right) \delta_{k m}-\alpha_{k} \alpha_{m}^{*} A(E)\right] \varphi_{m}(E)-\sum_{l} B(E) \beta_{k} \beta_{l}^{*} \varphi_{l} \\
=-c \sum_{m} f_{m k}^{*}\left(E-c_{m}\right),
\end{array}
$$

where $B(E)$ is

$$
B(E)=\sum_{m} \frac{\beta_{m}^{*} \beta_{m}}{c_{m}+\omega_{0}-E} .
$$

Equation (20) can be written in a more compact form as

$$
\sum_{s} \varphi_{s}(E)\left[\left(c_{k}-E\right) \delta_{k s}-\alpha_{k}^{*} \alpha_{s} A(E)-\beta_{k}^{*} \beta_{s} B(E)\right]=-c \sum_{l} f_{l k}^{*}\left(c_{l}-E\right)
$$

where the functions on the variable $E, A(E)$ and $B(E)$, do not depend either on $k$ and $s$. Equation (22) can be also written in matrix form:

$$
T\left(\begin{array}{c}
\cdots \\
\varphi_{s}(E) \\
\cdots
\end{array}\right)=\left(\begin{array}{c}
\cdots \\
-c \sum_{l} f_{l k}^{*}\left(c_{l}-E\right) \\
\cdots
\end{array}\right)
$$


where the entries in the matrix $T$ are given by

$$
\begin{aligned}
& T_{k s}=\left(c_{k}-E\right) \delta_{k s}-\alpha_{k}^{*} \alpha_{s} A(E)-\beta_{k}^{*} \beta_{s} B(E) \\
= & \left(c_{k}-E\right)\left\{\delta_{k s}-\frac{\alpha_{k}^{*} \alpha_{s} A(E)}{\left(c_{k}-E\right)}-\frac{\beta_{k}^{*} \beta_{s} B(E)}{\left(c_{k}-E\right)}\right\} .
\end{aligned}
$$

To some extent, we have borrowed the notion frequently encountered in Theory of Reactions (see Newton [13]) in dealing with the formal solution of systems of coupled equations, where $T$ stands for the transfer matrix. A solution to our problem with the choice (16) can be obtained provided that $\mathrm{T}$ be invertible. To prove this claim, we use the matrix identity $(1-A)^{-1}=\sum_{n} A^{n}$. In consequence, the entries of $T^{-1}$ are the following:

$$
\begin{array}{r}
\left(T^{-1}\right)_{k s}=\frac{1}{c_{k}-E}\left\{\delta_{k s}-\frac{\alpha_{k}^{*} \alpha_{s} A(E)}{c_{k}-E}-\frac{\beta_{k}^{*} \beta_{s} B(E)}{c_{k}-E}\right\}^{-1} \\
=\frac{1}{c_{k}-E}\left\{\delta_{k s}+\left(\frac{\alpha_{k}^{*} \alpha_{s} A(E)}{c_{k}-E}+\frac{\beta_{k}^{*} \beta_{s} B(E)}{c_{k}-E}\right)\right. \\
\left.+\left(\frac{\alpha_{k}^{*} \alpha_{k^{\prime}} A(E)}{c_{k}-E}+\frac{\beta_{k} \beta_{k^{\prime}} B(E)}{c_{k}-E}\right)\left(\frac{\alpha_{k^{\prime}}^{*} \alpha_{s} A(E)}{c_{k^{\prime}}-E}+\frac{\beta_{k^{\prime}} \beta_{s} B(E)}{c_{k^{\prime}}-E}\right)+\cdots\right\} \\
=\frac{\delta_{k s}}{c_{k}-E}+\alpha_{k}^{*} \alpha_{s} a_{k s}+\beta_{k}^{*} \beta_{s} b_{k s}+\alpha_{k}^{*} \beta_{s} c_{k s}+\beta_{k}^{*} \alpha_{s} d_{k s} .
\end{array}
$$

Then, it remains to determine the coefficients $a_{k s}, b_{k s}, c_{k s}, d_{k s}$, with the use of the obvious relation $\sum_{k^{\prime}} T_{k k^{\prime}}^{-1} T_{k^{\prime} s}=\delta_{k s}$, which gives a expression of the form:

$$
\begin{aligned}
\alpha_{k}^{*} \alpha_{s} C_{1}\left(a_{k s}, b_{k s}, \ldots\right)+\beta_{k}^{*} \beta_{s} C_{2}\left(a_{k s}, b_{k s}, \ldots\right) & +\alpha_{k}^{*} \beta_{s} C_{3}\left(a_{k s}, b_{k s}, \ldots\right) \\
& +\beta_{k}^{*} \alpha_{s} C_{4}\left(a_{k s}, b_{k s}, \ldots\right)=0 .
\end{aligned}
$$

If we assume linear independence of the variables $\alpha_{k}$ and $\beta_{s}$, the coefficients of (26) should vanish for all values of the indices $r$ and $s$ : 


$$
C_{p}\left(a_{k s}, b_{k s}, \ldots\right)=0, \quad p=1,2,3,4 .
$$

By implementing in (25) condition (26) one obtains

$$
\begin{aligned}
&\left(c_{s}-E\right) a_{k s}-\frac{A(E)}{c_{k}-E}-A(E) \sum_{k^{\prime}}\left[\alpha_{k^{\prime}} \alpha_{k^{\prime}}^{*} a_{k^{\prime} s}+\beta_{k^{\prime}} \alpha_{k^{\prime}}^{*} c_{k k^{\prime}}\right]=0 . \\
&\left(c_{s}-E\right) b_{k s}-\frac{B(E)}{c_{k}-E}-B(E) \sum_{k^{\prime}}\left[\beta_{k^{\prime}} \beta_{k}^{*} b_{k k^{\prime}}+\alpha_{k^{\prime}} \beta_{k^{\prime}}^{*} d_{k k^{\prime}}\right]=0 . \\
& c_{k s}\left(c_{s}-E\right)-B(E) \sum_{k^{\prime}}\left[\alpha_{k^{\prime}} \beta_{k^{\prime}}^{*} a_{k k^{\prime}}+\beta_{k^{\prime}} \beta_{k^{\prime}}^{*} c_{k k^{\prime}}\right]=0 . \\
& d_{k s}\left(c_{s}-E\right)-A(E) \sum_{k^{\prime}}\left[\beta_{k^{\prime}} \alpha_{k^{\prime}}^{*} b_{k k^{\prime}}+\alpha_{k^{\prime}} \alpha_{k^{\prime}}^{*} d_{k k^{\prime}}\right]=0 .
\end{aligned}
$$

Let us start by solving Equation (29). It can be written as

$$
c_{k s}=\frac{B(E) R_{k}}{c_{s}-E}
$$

where

$$
R_{k}=\sum_{k^{\prime}}\left[\alpha_{k^{\prime}} \beta_{k^{\prime}}^{*} a_{k k^{\prime}}+\beta_{k^{\prime}} \beta_{k^{\prime}}^{*} c_{k k^{\prime}}\right]
$$

In order to simplify subsequent equations, we shall introduce the following notation:

$$
\tau(\alpha, \beta, E):=\sum_{k} \frac{\alpha_{k} \beta_{k}}{c_{k}-E} .
$$

Using this definition in (31, 32) gives

$$
\frac{B(E) R_{k}}{c_{s}-E}=\frac{B(E)}{c_{s}-E}\left(\sum_{k^{\prime}} \alpha_{k^{\prime}} \beta_{k^{\prime}}^{*} a_{k k^{\prime}}+\tau\left(\beta, \beta^{*}, E\right) B(E) R_{k}\right),
$$

and from it the quantity $R_{k}$ can be expressed as 


$$
R_{k}=\frac{\sum_{k^{\prime}} a_{k^{\prime}} \beta_{k^{\prime}}^{*} a_{k k^{\prime}}}{1-B(E) \tau\left(\beta, \beta^{*}, E\right)}
$$

In a similar manner

$$
c_{k s}=\frac{B(E)}{c_{s}-E} \sum_{k^{\prime}} \frac{\alpha_{k^{\prime}} \beta_{k^{\prime}}^{*} a_{k k^{\prime}}}{1-B(E) \tau\left(\beta, \beta^{*}, E\right)} .
$$

and

$$
a_{k s}=\frac{A(E)}{c_{s}-E}\left[\frac{1}{c_{k}-E}+\sum_{k^{\prime}}\left(\alpha_{k^{\prime}} \alpha_{k^{\prime}}^{*} a_{k k^{\prime}}+\beta_{k^{\prime}} \alpha_{k^{\prime}} c_{k k^{\prime}}\right)\right] .
$$

Note that the inverse matrix $T^{-1}$ does exist if and only if the $R_{k}$ are nonsingular for all $E$, i.e., the denominator in (35) does not vanish:

$$
1-B(E) \tau\left(\beta, \beta^{*}, E\right) \neq 0 .
$$

If one proceeds with the expression of $a_{k s}$ in (27) as done with $c_{k s}$, one finds

$$
a_{k s}=\frac{A(E) \Pi_{k}}{c_{s}-E}
$$

with

$$
\Pi_{k}:=\frac{1}{c_{k}-E}+\sum_{k^{\prime}} \alpha_{k^{\prime}} \alpha_{k^{\prime}}^{*} a_{k k^{\prime}}+\frac{B(E) \tau\left(\beta, \alpha^{*}, E\right)}{1-B(E) \tau\left(\beta, \beta^{*}, E\right)} \sum_{k^{\prime}} \alpha_{k^{\prime}} \beta_{k^{\prime}}^{*} a_{k k^{\prime}} .
$$

From (39) and (40), one gets

$$
\begin{array}{r}
\frac{A(E) \Pi_{k}}{c_{s}-E}=\frac{A(E)}{c_{s}-E}\left[\frac{1}{c_{k}-E}+A(E) \Pi_{k} \tau\left(\alpha, \alpha^{*}, E\right)\right. \\
\left.+B(E) \frac{\tau\left(\beta, \alpha^{*}, E\right) \tau\left(\alpha, \beta^{*}, E\right)}{1-\tau\left(\beta, \beta^{*}, E\right) B(E)}+A(E) \Pi_{k}\right],
\end{array}
$$

from which we obtain a consistent equation for $\Pi_{k}$ :

$$
\Pi_{k}=\frac{1}{c_{k}-E}\left[1-A(E) \tau\left(\alpha, \alpha^{*}, E\right)-A(E) B(E) \frac{\tau\left(\beta, \alpha^{*}, E\right) \tau\left(\alpha, \beta^{*}, E\right)}{1-\tau\left(\beta, \beta^{*}, E\right) B(E)}\right]
$$


leading to

$$
a_{k s}=\frac{A(E)\left(1-B(E) \tau\left(\beta, \beta^{*}, E\right)\right)}{\left(c_{k}-E\right)\left(c_{s}-E\right)} \Delta^{-1}
$$

with

$$
\begin{array}{r}
\Delta:=1-A(E) \tau\left(\alpha, \alpha^{*}, E\right)-B(E) \tau\left(\beta, \beta^{*}, E\right) \\
+A(E) B(E)\left[\tau\left(\alpha, \alpha^{*}, E\right) \tau\left(\beta, \beta^{*}, E\right)-\tau\left(\alpha, \beta^{*}, E\right) \tau\left(\beta, \alpha^{*}, E\right)\right] .
\end{array}
$$

Accordingly, nontrivial solutions of $a_{k s}\left(a_{k s} \neq 0\right)$ exist if and only if $\Delta \neq 0$ for all values of the energy $E$.

Finally, the expressions for the remaining coefficients are

$$
\begin{gathered}
b_{k s}=\frac{B(E)\left(1-A(E) \tau\left(\alpha, \alpha^{*}, E\right)\right)}{\left(c_{s}-E\right)\left(c_{k}-E\right)} \Delta^{-1}, \\
d_{k s}=\frac{A(E) B(E) \tau\left(\beta, \alpha^{*}, E\right)}{\left(c_{s}-E\right)\left(c_{k}-E\right)} \Delta^{-1} .
\end{gathered}
$$

With these elements, we are now in the position of writing the explicit form for the inverse matrix $T^{-1}$

$$
\begin{array}{r}
T_{k s}^{-1}=\frac{\delta_{k s}}{c_{k}-E}+\frac{\Delta^{-1}}{\left(c_{k}-E\right)\left(c_{s}-E\right)}\left\{\alpha_{k}^{*} \alpha_{s} A(E)\left[1-B(E) \tau\left(\beta, \beta^{*}, E\right)\right]\right. \\
+\beta_{k}^{*} \beta_{s} B(E)\left[1-A(E) \tau\left(\alpha, \alpha^{*}, E\right)\right]+\alpha_{k}^{*} \beta_{s}^{*} A(E) B(E) \tau\left(\alpha, \beta^{*}, E\right) \\
\left.+\beta_{s}^{*} \alpha_{s} A(E) B(E) \tau\left(\beta, \alpha^{*}, E\right)\right\} .
\end{array}
$$

In consequence, the formal solution of Equation (23) is given by

$$
\varphi_{k}(E)=-c \sum_{k} T_{k n}^{-1}\left(\sum_{l} f_{l n}^{*}\left(c_{l}-E\right)\right)
$$

and

$$
\phi_{k 1}(E)=c \frac{\beta_{k}}{c_{k}+\omega_{0}-E} \sum_{n, m} \beta_{m}^{*} T_{m n}^{-1}\left(\sum_{l} f_{l k}^{*}\left(c_{l}-E\right)\right) .
$$


Thus, inserting (48) and (49) in (13) and then in (8) the solution to our problem is completely determined. Note that the structure of the solutions is preserved by form factors which may not been separable as those of the choice (16), since they are absorbed in the definition of the functions $\tau$.

\section{Concluding remarks.}

In this paper, we have described the solutions of the extended Friedrichs model. The proposed model includes fermion-boson couplings in addition to the boson-boson couplings. From the physical point of view, this extension may become a suitable tool for the treatment of decaying states which participate in the observable total decay width of composite nuclear systems. From the mathematical point of view, we have generalized the schematic solution proposed in a previous article [6] and formulated the solution in a more compact and formal way. The main features of the proposed method can be summarized as follows:

i.) The resonant behavior of the fermionic sector depends on the resonant structure of the boson field, which participate in the fermion solution via the fermion-boson coupling.

ii.) The set of coupled equations is greatly simplified by the use of a T-matrix like formalism, which allows us to identify the resonant behavior of the solutions.

Further applications of the set of Equations (47-49) to situations of physical interest are in progress.

\section{Acknowledgements.}

Financial support is acknowledged to the Ministry of Education of Spain, grants PR2004-0080, MTM2005-09183, FIS2005-03989, SAB2004-0169, the

Junta de Castilla y León Project VA013C05, the Russian Science Foundation Grant 04-01-00352 and the CONICET (Argentina).

\section{References}

[1] K. O. Friedrichs, Commun. Pure Appl. Math., 1, 361 (1948). 
[2] A.Likhoded, G.P. Pronko, Int. J. Theor. Phys., 36, 2335 (1997).

[3] I.E. Antoniou, M. Gadella, J. Mateo, G.P. Pronko, Int. J. Theor. Phys., 42, 2389 (2003).

[4] I.E. Antoniou, M. Gadella, J. Mateo, G.P. Pronko, J. Phys A: Math. Gen, 36, 12109 (2003).

[5] O. Civitarese, M. Gadella, Phys. Rep., 396, 41-113 (2004).

[6] O. Civitarese, M. Gadella, International Journal of Modern Physics E, 15, 1273-1290 (2006).

[7] A.T. Kruppa, P.H. Heenen, Phys. Rev. C, 63 (2001) 034304.

[8] G. Rudin, M. Gadella, Int. J. Quan. Chem., 58, 441 (1996).

[9] I.E. Antoniou, M. Gadella, Irreversibility, Resonances and Rigged Hilbert Spaces in Irreversible Quantum Dynamics, F. Benatti and R. Floreanini, Eds., Springer Lecture Notes in Physics, vol 622, Springer Verlag, Berlin, Heidelberg (2003), pp. 246-302.

[10] H. Baumgärtel, Reviews in Mathematical Physics, 18 (2006) 61.

[11] S. Weinberg, The Quantum Theory of Field. Volume I: Foundations, Cambridge University Press, Cambridge (1995).

[12] A. Bohr, B. Mottelson, Nuclear Structure, vol. II (Benjamin, Reading, M. A. 1975).

[13] R.G. Newton, Scattering Theory of Waves and Particles (Springer, New York, Heidelberg, Berlin, 1982).

[14] I.E. Antoniou, M. Gadella, I. Prigogine, G.P. Pronko, J. Math. Phys., 39, 2995 (1998).

[15] L. Horwitz, P. Marchand, Rocky Mt. J. Math., 1, 225 (1971).

[16] I.E. Antoniou, M. Gadella, G.P. Pronko, J. Math. Phys., 39, 2459-2475 (1998).

[17] O. Civitarese, A.G. Dumrauf, R.J. Liotta, Phys. Rev. C, 47, 1463 (1993). 